\title{
Dimensionamento de Reservatório de Detenção partindo da Relação IDF regente no Município de Fortaleza (CE)
}

\author{
Anísio de Sousa Meneses Filho' ${ }^{\prime}$ Lucas Florêncio da Cunha Teixeira2; Gustavo Siebra Lopes ${ }^{l}$ \\ \anisiomeneses@unifor.br
}

1. Universidade de Fortaleza - Fortaleza (CE).

2. Universidade Federal do Ceará - Fortaleza (CE).

\author{
Histórico do Artigo: \\ Recebido em: 04 de maio de $2020 \quad$ Aceito em: 29 de junho de $2020 \quad$ Publicado em: 31 de dezembro de 2020
}

\begin{abstract}
Resumo: 0 presente trabalho objetivou propor um critério simples para a estimativa no dimensionamento de volume para reservatório de detenção considerando diferentes períodos de retorno e cenários ocupacionais, partindo da relação IDF (intensidade-duração-frequência) de eventos críticos de precipitação para o município de Fortaleza, com a apresentação de formulações matemáticas de regressão que relacionam essas variáveis. Foi feita uma fundamentação teórica embasando os problemas relacionados à urbanização no ciclo hidrológico natural, além de relacionar as variáveis necessárias para o dimensionamento expedito. Fez-se uma abordagem acerca de reservatório de detenção, expondo suas características e locais onde pode ser aplicado e seu pré-dimensionamento. Na metodologia, adotou-se o método da "curva envelope" para estimar o volume excedente que foi utilizado para 0 dimensionamento do reservatório de detenção. Utilizou-se o software MATLABß para resolver a equação resultante do método, obtendo-se 60 tempos de duração do evento e 60 alturas de lâmina d'água, realizando a combinação de 10 taxas ocupacionais e 6 períodos de retorno. Por exemplo, para uma área de contribuição de 40 hectares, área impermeável de $70 \%$ e recorrência de 25 anos, o reservatório de detenção deve ter capacidade da ordem de 18.000 $\mathrm{m}^{3}$ para atender a vazão de pré-dimensionamento assumida. 0 resultado deste trabalho se mostrou potencialmente útil para a fase de planejamento dos projetos de drenagem urbana em diversas áreas de Fortaleza, variando-se a taxa ocupacional e o período de retorno conforme desejado pelo projetista, podendo ser adaptada para outras localidades desde que disponha de suas respectivas relações IDF.

Palavras-chave: Drenagem urbana, Técnicas compensatórias, Urbanização, Planejamento.
\end{abstract}

\section{Sizing of Detention Reservoir based on the IDF Relationship of Fortaleza (CE)}

Abstract: This paper aimed at proposing a simple criterion for estimates on volume sizing for detention reservoir considering different return periods and occupational scenarios, based on Fortaleza city's IDF relationship (intensity-duration-frequency) of critical rainfall events, presenting mathematical regression formulations linking those variables. A theoretical background was made to support problems related to urbanization in the natural hydrologic cycle, and relating all needed variables for the expeditious sizing. Thus, the detention reservoir was detailed, with its characteristics and places where it can be applied and its pre-sizing method being explained. 0n the methodology, the envelope curve method was adopted to estimate the exceeding volume which was used for sizing the detention reservoir. Software MATLAB ${ }^{\circledR}$ was used to solve the resulting equation, yielding 60 durations and 60 water line heights, from a combination of 10 occupational rates and 6 return periods. For example, for a contribution area of 40 hectares, impervious area of $70 \%$ and return period of 25 years, the detention reservoir needs the capacity of about $18.000 \mathrm{~m}^{3}$ to meet the assumed pre-sizing flow rate. This paper's result showed up to be potentially useful for the urban drainage design planning phase in several areas in Fortaleza city, in which the occupational rate and the return period can be varied according to the designer's will. In addition to that, this method can be adapted to other areas once their IDF curve is available.

Keywords: Urban drainage, Best management practices, Urbanization, Planning. 


\title{
Dimensionamiento de la Cuenca de Detención a partir de la curva IDF de Fortaleza (CE)
}

\begin{abstract}
Resumen: El objetivo de este trabajo fue proponer un criterio sencillo para la estimación del dimensionamiento de volumen para cuenca de detención considerando diferentes períodos de retorno y escenarios ocupacionales, a partir de la curva IDF (intensidad-duración-frecuencia) de eventos críticos de precipitación para Fortaleza, con la presentación de formulaciones de regresión matemática que correlacionan estas variables. Así, se presentó una base teórica basando los problemas relacionados con la urbanización en el ciclo hidrológico natural, además de enumerar las variables que son necesarias para el dimensionamiento expedito. Se hizo un acercamiento sobre la cuenca de detención, exponiendo sus características y lugares donde se puede aplicar y su pre-dimensionamiento. En la metodología, se adoptó el método de "curva envolvente" para estimar el volumen excedente que se utilizó para el dimensionamiento de la cuenca de detención. El software MATLABß fue empleado para solucionar la ecuación resultante del método, obteniendo 60 duraciones y 60 profundidades de água. Por ejemplo, para un área de contribución de 40 hectáreas, un área impermeable del $70 \%$ y un período de retorno de 25 años, la cuenca de detención necesita una capacidad de aproximadamente $18.000 \mathrm{~m}^{3}$ para cumplir con la tasa de flujo de predimensionamiento supuesta. El resultado de este trabajo se mostró potencialmente conveniente para la fase de planificación de proyectos de drenaje urbano en diferentes áreas de Fortaleza, variando la tasa ocupacional y el período de retorno según lo deseado por el diseñador, y puede adaptarse a otros sitios siempre que tengan sus respectivas curvas IDF.

Palabras clave: Drenaje urbano, Técnicas compensatorias, Urbanización, Planificación.
\end{abstract}

\section{INTRODUÇÃO}

0 problema das cheias urbanas assola praticamente todas as cidades que tiveram uma dinâmica ocupacional desordenada. Essa situação implica a necessidade de intervenções antrópicas para mitigar os dramas sociais e as perdas econômicas advindas das precipitações críticas que se convertem em grandes volumes para o escoamento superficial direto.

No decorrer dos anos, nota-se um aumento na frequência de eventos críticos que envolvem enchentes ou alagamentos em áreas urbanas, muitas vezes associados a alterações inadequadas no sistema natural de macrodrenagem. Para Bahiense (2013), esses eventos são, de forma invariável, críticos e trazem graves consequências à maior parte das cidades desenvolvidas sem o planejamento adequado ou, ainda, sem a sua devida implementação. Assim, as cheias urbanas podem ser consideradas um dos principais problemas das atuais cidades, afetando aspectos ambientais, sociais, econômicos e pondo em risco a vida humana.

Isso se dá pelo processo de urbanização acompanhado de falta de controle do uso e ocupação do solo, que resulta em impermeabilização de áreas que passam a contribuir para maior geração de escoamento superficial. Há a necessidade, portanto, de enfrentamento dos impactos gerados pela urbanização.

As abordagens clássicas de manejo de águas pluviais costumam levar em consideração o dimensionamento e a manutenção dos dispositivos componentes dos sistemas de micro e 
Dimensionamento de Reservatório de Detenção partindo da Relação IDF regente no Município de Fortaleza (CE)

macrodrenagem. 0 principal objetivo, para essa abordagem, é o rápido escoamento das águas pluviais.

As técnicas mais recentes de drenagem urbana costumam englobar soluções alternativas, incluindo a integração de diversos propósitos. Segundo Souza, Medeiros e Miranda (2017), o desenvolvimento sustentável é favorecido pelo uso dessas técnicas compensatórias, pelas quais se buscam resgatar as condições de pré-urbanização, assim como permitem o controle de volume e velocidade de escoamento superficial durante as precipitações. Assim, essas técnicas buscam compensar ou mitigar os efeitos da urbanização sobre as bacias hidrográficas.

Dentre as técnicas compensatórias existentes, foi selecionado para a exploração neste trabalho o reservatório ou bacia de detenção, que, para Bahiense (2013), consiste em uma estrutura de armazenamento temporário que pode direcionar a água ao sistema de drenagem ou a outro reservatório, sendo sua principal função a acumulação de escoamento excedente gerado pela impermeabilização, deixando escoar através orifícios ou vertedores uma vazão similar à existente na condição natural do terreno.

Este trabalho se destina a orientar o pré-dimensionamento do reservatório de detenção a partir da relação IDF de Fortaleza, considerando diferentes períodos de retorno e cenários ocupacionais. Para isso, são propostas relações matemáticas de regressão que permitem uma estimativa preliminar da capacidade dos dispositivos de armazenamento temporário para 0 controle de cheias urbanas.

\section{Impactos hidrológicos da urbanização}

A bacia hidrográfica, no seu aspecto natural, geralmente apresenta um comportamento equilibrado e harmônico, com bosques protegendo o escoamento em encostas, mata ciliar protegendo as margens dos rios e processos de infiltração que permitem a recarga dos lençóis freáticos, garantindo, assim, a vazão de base. Com o advento da urbanização, entretanto, as bacias hidrográficas sofrem alterações em seu ciclo hidrológico natural e, portanto, geram respostas fluviais diferentes. (MIGUEZ; VERÓL; REZENDE, 2015 citado por TEIXEIRA, 2017).

Com o avanço do processo, há a necessidade de se pavimentar áreas consideravelmente extensas, impactando diretamente em maior geração de escoamento superficial. Todas essas mudanças no ciclo hidrológico geram um desequilíbrio no balanço hídrico que, combinado com outros fatores, como a maior geração de resíduos sólidos, deterioram a qualidade de vida da 
população, com aumento significante da frequência e nível das inundações, redução de qualidade da água e aumento de materiais sólidos nos corpos receptores (CURITIBA, 2002).

Na Figura 1 a seguir ilustram-se os hidrogramas gerados pela bacia em seu estado natural e o resultante do processo de urbanização.
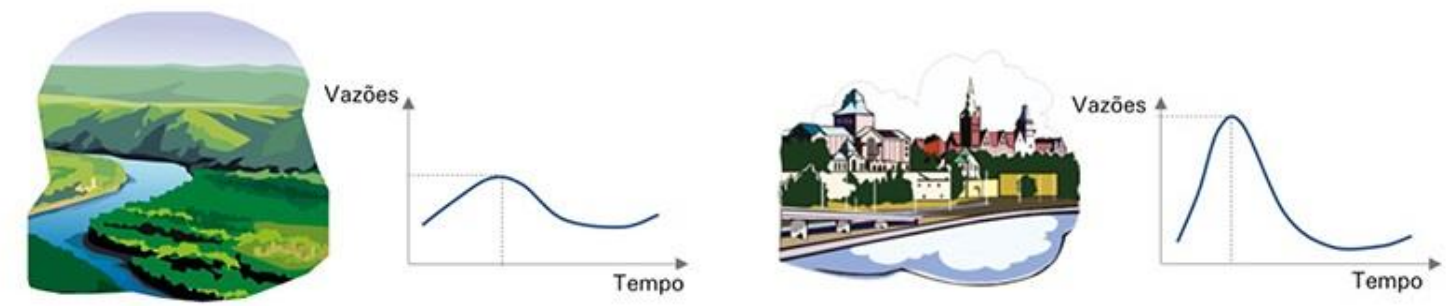

Figura 1. Impactos no hidrograma decorrentes do processo de urbanização Fonte: Miguez, Veról e Rezende, 2015, adaptado, p. 7.

Observando a figura acima, pode-se inferir que para a situação natural, há menores picos de cheia, menores volumes escoados, maior tempo de concentração e maior vazão de base, uma vez que ocorre maior infiltração. Na situação contrária da bacia urbanizada, percebem-se maiores picos de cheia, maiores volumes de escoamento superficial que ocasionam menor tempo de concentração e, além disso, menor vazão de base devido à menor infiltração.

\section{Precipitações intensas}

Tendo em vista que, durante a criação e ampliação de cidades, são promovidas alterações no ciclo hidrológico natural, surge a necessidade de conceber e implantar um sistema de drenagem das águas pluviais, em escala reduzida (microdrenagem) e coletando a água pluvial de todo o centro urbano (macrodrenagem).

No entanto, observa-se que, em grande parte dos centros urbanos brasileiros, esses sistemas apresentam deficiências, causando transtornos aos habitantes dos municípios, como o congestionamento de veículos e pessoas. A situação é agravada pela incidência de precipitações de grande intensidade, que podem gerar perdas humanas e materiais. Esse tipo de problema pode resultar de uma má concepção, em que o risco assumido não levou em consideração a ocorrência de maiores precipitações na região, ou de má conservação dos componentes do sistema, não observando manutenções corretivas e/ou preventivas, além do acúmulo de resíduos sólidos que obstruem as seções dimensionadas para a condução adequada da água.

\section{Período de retorno}


Dimensionamento de Reservatório de Detenção partindo da Relação IDF regente no Município de Fortaleza (CE)

Uma das variáveis envolvidas no dimensionamento que ajuda na prevenção dos problemas relacionados é o período de retorno $(T R)$, que corresponde ao tempo médio que determinado evento hidrológico pode ser igualado ou superado.

Associado a essa variável, está o risco hidrológico, que reflete na possibilidade de ruptura de determinada obra. Dessa forma, o risco relaciona a probabilidade que ocorra um evento para determinado $T R$ (em anos) ocorra durante um intervalo de tempo $n$ (em anos), conforme Equação l:

$J=1-\left(1-\frac{1}{T R}\right)^{n}$

Quanto menor o risco assumido, maior será o período de retorno. Esse parâmetro, portanto, incide diretamente na durabilidade e segurança das obras. A redução do risco hidrológico implica intervenções de maior magnitude e, consequentemente, maiores custos para o empreendimento da estrutura hidráulica. Em projeto, a escolha do $T R$ está condicionada ao nível de risco aceitável ante os impactos decorrentes do colapso ou do não atendimento do propósito da intervenção. De forma geral, adotam-se elevados períodos de retorno para usinas hidrelétricas, por exemplo, enquanto para sistemas de drenagem urbana, para Bidone e Tucci (1995, p. 87), esse valor costuma ser menos elevado, variando de 2 a 100 anos, dependendo da importância da área.

\section{Relação IDF (intensidade-duração-frequência)}

Sabendo-se o valor do período de retorno que será adotado para um sistema de drenagem, pode-se estimar a quantidade de chuva que irá precipitar ao longo do tempo a partir da formulação matemática da relação IDF, que, para Teixeira (2017), requer estudos de vários anos e comparação de dados pluviográficos.

Outro parâmetro que precisa ser adotado para utilizar a relação IDF é o tempo de duração crítica do evento chuvoso, que deve ser longa o suficiente para que toda a bacia contribua para o escoamento superficial. Nesse caso, um critério compatível com pequenas áreas de contribuição é que a precipitação efetiva deve ter a mesma duração do tempo de concentração da bacia contribuinte para o trecho a ser dimensionado (TEIXEIRA, 2017).

De forma genérica, as curvas IDF podem ser escritas como:

$i=\frac{a \cdot T R^{b}}{(t+c)^{d}}$ 
Sendo $a, b, c$ e $d$ parâmetros que variam para cada região, $i$ a intensidade de chuva, $T R$ o período de retorno e $t$ o tempo de duração da chuva.

No caso de Fortaleza (CE), a equação mais recente foi elaborada por Silva, Palácio Júnior e Campos (2012), que analisaram 30 anos de registros de chuva e 611 hietogramas da estação climatológica da Universidade Federal do Ceará, utilizando durações de 5, 10, 20, 30, 45, 60 e 120 minutos. A relação IDF de Fortaleza é a Equação 3, sendo $i$ em mm/h, $T R$ em anos e $t$ em min: $i=\frac{2.345,29 \cdot T R^{0,173}}{(t+28,31)^{0,904}}$

Aplicando a Equação 3, na Figura 2 é demonstrado o aspecto característico de uma relação IDF com os valores de intensidade e duração.

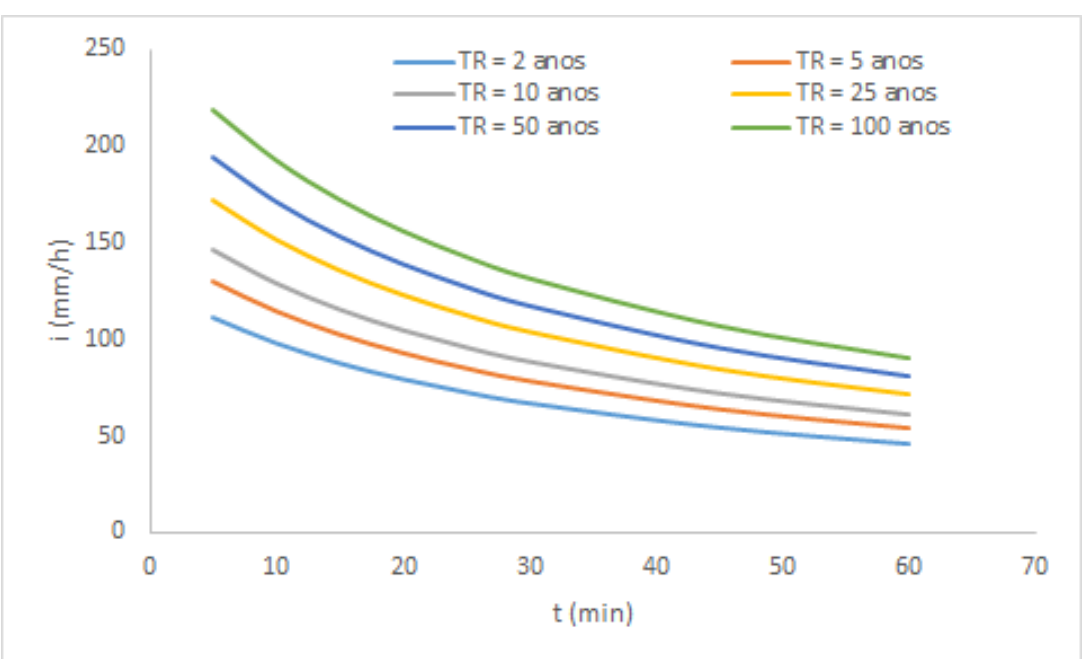

Figura 2. Aspecto característico da relação IDF Fonte: Elaborado pelos autores, 2020.

Observa-se que a intensidade de chuva diminui à medida em que se aumenta a duração da chuva e, além disso, maiores períodos de retorno geram maiores intensidades de chuva.

\section{Curva PDF (precipitação-duração-frequência)}

Uma outra forma de apresentação da curva característica da precipitação é através da curva PDF, que contempla as precipitações acumuladas e se associa à integração da relação IDF assumindo uma intensidade média constante para uma dada duração do evento.

Segundo Baptista, Nascimento e Barraud (2005, p. 109), as equações seguintes aproximam as curvas IDF:

$$
\begin{aligned}
& i(t, T R)=a(T R) t b(T R) \\
& i(t, T R)=a(T R) /(t+b(T R))
\end{aligned}
$$


Dimensionamento de Reservatório de Detenção partindo da Relação IDF regente no Município de Fortaleza (CE)

$$
i(t, T R)=a(T R)(t+b(T R))^{c(T R)}
$$

Sendo $i$ a intensidade de chuva, TR o período de retorno, $t$ a duração da chuva e $a, b$ e c parâmetros que variam para cada região.

Para o dimensionamento de obras de detenção, é necessário transformar as intensidades em alturas precipitadas, sendo estas o produto da duração pela intensidade.

Aplicando na relação IDF os mesmos valores da Figura 2, pode ser montada a curva PDF, demonstrando o seu aspecto característico, conforme Figura 3.

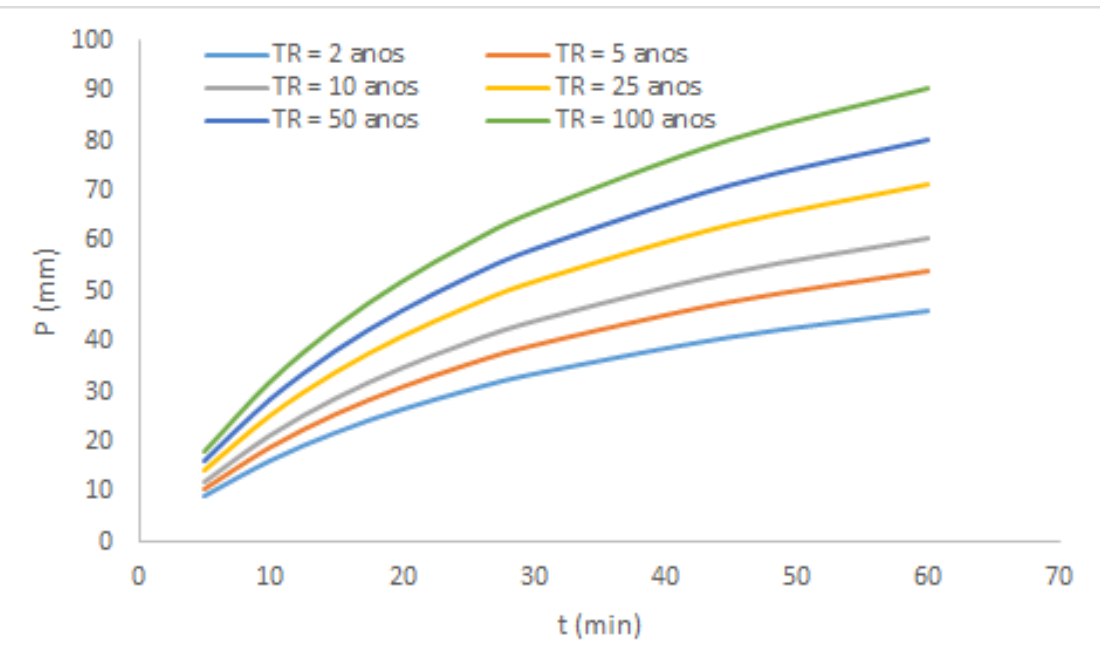

Figura 3. Aspecto característico da curva PDF

Fonte: Elaborado pelos autores, 2020.

\section{Estimativa da vazão pelo Método Racional}

Para a estimativa da vazão máxima gerada pela bacia hidrográfica, aplica-se o Método Racional que, para Tucci (1993) citado por Teixeira (2017), é largamente utilizado para pequenas bacias, englobando todos os processos no coeficiente de escoamento superficial.

Os princípios básicos da metodologia do Método Racional são:

- Considera a duração de precipitação intensa igual ao tempo de concentração. Assim, já que a duração é inversamente proporcional à intensidade da chuva, admite-se que a bacia é suficientemente pequena para que essa situação ocorra;

- $\quad$ Adota um coeficiente único de perdas, o coeficiente de escoamento superficial (C), estimado com base nas características da bacia;

- Não avalia o volume da cheia e a distribuição temporal de vazões. 
0 método pressupõe que o tempo de pico do hidrograma triangular unitário é igual ao tempo de duração da chuva que corresponde ao tempo de concentração da bacia. Nesse instante, toda a bacia contribui para a vazão, que tem o seu pico no final da duração da chuva. Na Figura 4 são exibidos o hidrograma unitário triangular, representado pelas linhas azuis, e o hietograma da chuva, representado pelas linhas laranjas, ilustrando essa situação descrita, onde $Q_{P}$ e $t_{P}$ representam a vazão de pico e o tempo de pico do hidrograma, respectivamente, e $P$ representa a precipitação do hietograma.

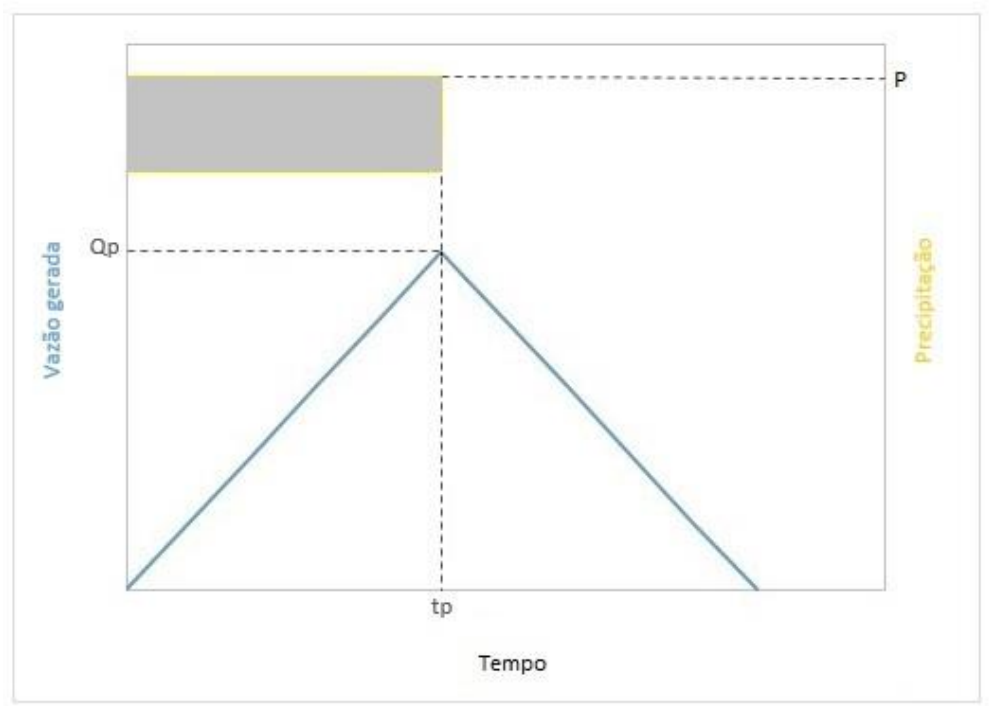

Figura 4. Situação pressuposta para aplicação do Método Racional Fonte: Elaborado pelos autores, 2020.

A formulação básica do Método Racional é a Equação 7:

$Q=2,778 \cdot C \cdot i \cdot A$

Sendo $Q$ a vazão máxima em $\mathrm{L} / \mathrm{s}, C$ o coeficiente de escoamento superficial, $i$ a intensidade de chuva em $\mathrm{mm} / \mathrm{h}$ e $A$ a área da bacia de drenagem em hectares (ha).

\section{Técnicas compensatórias em drenagem urbana}

Nos sistemas convencionais de drenagem, a água proveniente das chuvas é captada e conduzida por condutos artificiais, em geral subterrâneos, funcionando pela ação da gravidade de forma a serem evacuadas dos centros urbanos e conduzidos a corpos hídricos rapidamente. Entretanto, foram surgindo novas abordagens no tocante a soluções de manejo de águas pluviais, que são as técnicas compensatórias. (MIGUEZ; VERÓL; REZENDE, 2015).

Segundo Secretaria Nacional de Saneamento Ambiental (2007), essas técnicas costumam compensar os efeitos da urbanização de forma sistemática, controlando na fonte o excedente de água gerado pela impermeabilização de áreas através de infiltração, evitando o seu 
Dimensionamento de Reservatório de Detenção partindo da Relação IDF regente no Município de Fortaleza (CE)

escoamento rápido para jusante, utilizando, para tanto, estruturas de armazenamento temporário.

As técnicas compensatórias se classificam segundo a posição em que sua estrutura é implantada em relação à área drenada pela mesma. Por essa classificação, são divididas em:

- $\quad$ Técnicas para controle na fonte: valas e valetas, trincheiras, poços;

- Técnicas para controle no sistema viário: pavimentos de concreto permeável, de asfalto permeável, de blocos vazados, intertravados, de alvenaria poliédrica;

- Técnicas para controle a jusante: reservatórios ou bacias de detenção, bacias de infiltração.

\section{Reservatório de detenção}

Segundo Curitiba (2002), o reservatório de detenção pode ser aplicado junto ao sistema de macrodrenagem da cidade. No âmbito de loteamentos, visa ampliar o controle de geração de escoamento superficial restrito a estes, assim como aliviar o sistema de macrodrenagem a jusante. Junto aos cursos d'água, objetivam o controle de cheias, sendo ferramenta importante para o planejamento da bacia como um todo. Destaca-se, de forma geral, como principal benefício da implantação desse mecanismo a atenuação do pico de vazão (laminação) e o retardo do tempo de pico, dois fatores fundamentais para o controle de enchentes urbanas. Por outro lado, algumas restrições podem ser reconhecidas no tocante à disponibilização de espaço físico conveniente e aos custos de aquisição de terreno, além de sua integração a outros equipamentos do sistema urbano. Operacionalmente, cabe reconhecer a necessidade de manutenção dos dispositivos hidráulicos de saída do reservatório para assegurar seu efetivo desempenho na laminação da cheia.

Em relação a aspectos construtivos do reservatório de detenção, estes dependem da área disponibilizada para a sua construção. Em regiões densamente ocupadas, opta-se por sua construção em paralelo (off-line), capaz de armazenar volumes maiores pela possibilidade de ser mais profundo que o leito do córrego. Quando há espaço disponível, a opção é a sua construção em série (in-line) com o leito do rio. (ABCP, 2013). 
A representação esquemática de um reservatório de detenção off-line é demonstrada na Figura 5.

Figura 5. Reservatório de detenção off-line

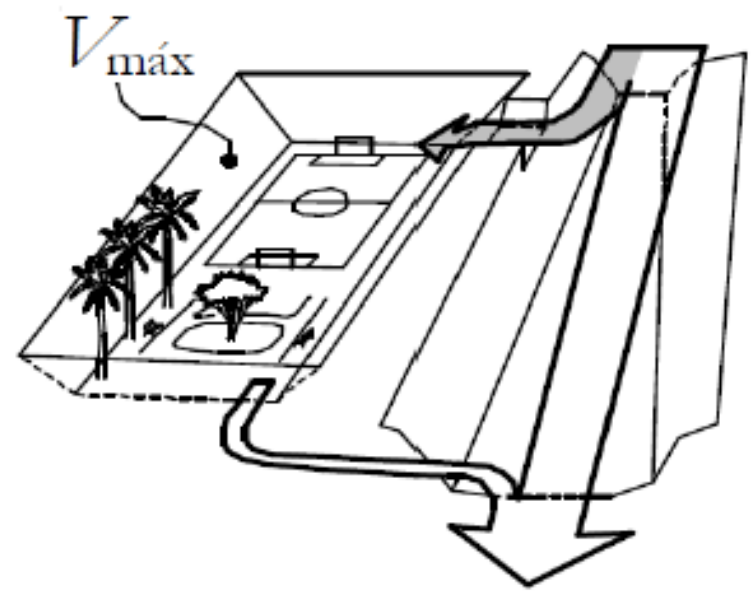
Fonte: Silveira e Goldenfum, 2007.

Uma das vantagens atribuíveis ao reservatório de detenção é que ele pode assumir múltiplos propósitos. Além do controle de cheias, esses equipamentos podem servir para práticas esportivas durante o período seco.

\section{Pré-dimensionamento do reservatório de detenção}

Segundo Silveira e Goldenfum (2007), o pré-dimensionamento do reservatório de detenção corresponde ao volume máximo a ser armazenado sobre toda a bacia contribuinte, segundo a Equação 8:

$$
V_{\text {máx }}=\left(\sqrt{\frac{a}{60}} \cdot \sqrt{C} \cdot T R^{b / 2}-\sqrt{\frac{c}{60}} \cdot \sqrt{q_{s}}\right)^{2}
$$

Onde $V_{\text {máx }}$ é o volume de acumulação em mm, $C$ é o coeficiente de escoamento, TR é 0 período de retorno em anos, $q_{s}$ é a vazão de saída em $\mathrm{mm} / \mathrm{h}$ e $a$ e $c$ são parâmetros que dependem da área onde está sendo aplicada a equação. Note-se que o volume de acumulação aqui referido se expressa em lâmina d'água, tendo em vista a relativização da volumetria pela área. Para se obter esse resultado $\mathrm{em} \mathrm{m}^{3}$ ha, multiplica-se 0 valor obtido por 10.

A partir desse ponto, pode-se estimar a área inundada e o volume absoluto necessário. Há três alternativas de pré-dimensionamento:

- Reservatório de detenção com leito impermeável;

- Reservatório de detenção com leito permeável e esgotamento por infiltração; 
Dimensionamento de Reservatório de Detenção partindo da Relação IDF regente no Município de Fortaleza (CE)

- Reservatório de detenção com leito permeável e esgotamento simultâneo por infiltração do solo e por tubulação.

Para a primeira alternativa, é conveniente dimensionar o tubo de saída com a vazão de saída $q_{s}$ igual à vazão de pré-desenvolvimento, ou seja:

$q_{s}=q_{\text {pre }}$

$(9)$

Para a segunda alternativa, deve-se levar em consideração a condutividade hidráulica saturada do solo. Adota-se a Equação 10 nesse caso:

$q_{s}=\alpha \cdot K_{\text {sat }}$

Onde $q_{s}$ é a vazão de saída em L/s/ha, $\alpha$ é um coeficiente redutor devido à colmatação (costuma-se adotar 0 valor de 0,5$)$ e $K_{\text {sat }}$ é a condutividade hidráulica saturada do solo em $\mathrm{mm} / \mathrm{h}$ (determinada por ensaios de infiltração).

Já para a terceira alternativa, a vazão de saída será a soma da vazão de prédimensionamento com a vazão de infiltração:

$q_{s}=q_{\text {pre }}+\alpha \cdot K_{\text {sat }}$

No âmbito deste trabalho, será tratado o dimensionamento considerando reservatório de detenção com leito impermeável.

\section{METOD0LOGIA}

\section{Coeficiente de escoamento na área urbana}

Partindo da equação básica do Método Racional (Equação 7) e relativizando a vazão em termos de área, de forma a encontrar uma equação generalizada, chega-se em:

$q_{n}=\frac{Q}{A}=2,778 \cdot C \cdot i$

Onde $q_{n}$ é a vazão específica natural em L/s/ha.

0 coeficiente $C$ pode ser desmembrado em duas parcelas, sendo uma referente à área permeável da bacia e outra à área impermeável, resultando na Equação 13:

$C=\frac{C_{p} \cdot A_{p}+C_{i} \cdot A_{i}}{A_{T}}=C_{p} \frac{A_{p}}{A_{T}}+C_{i} \frac{A_{i}}{A_{T}}$

Sendo os subscritos $p$ e $i$ referentes às áreas permeáveis e impermeáveis, respectivamente, e o subscrito $T$ referente à área total da bacia. Assumindo que:

$A I=\frac{A_{i}}{A_{T}}$ 
Onde $A I$ representa a parcela da área impermeável em relação à área total, em termos percentuais.

Substituindo a Equação 14 na Equação 13, resulta-se em:

$C=C_{p}+\left(C_{i}-C_{p}\right) \cdot A I$

Baseando-se em trabalhos anteriores desenvolvidos por Schueler (1987), Urbonas et al. (1990) e Tucci (2000), apud Curitiba (2002), $C_{i}=0,95$ e $C_{p}=0,15$. Substituindo na Equação 15 , tem-se:

$C=0,15+0,80 \cdot A I$

A equação acima é válida para $0 \leq A I \leq 1$.

Utilizando $t=60 \mathrm{~min}, A I=0$ e $C_{p}=0,15$ nas Equações 3 e 12:

$q_{n}=2,778 \cdot 0,15 \cdot 40,832 \cdot T R^{0,173}=17,015 \cdot T R^{0,173}$

(17)

A adoção do tempo de duração de 60 minutos se pauta na hipótese preliminar de que as áreas urbanas de contribuição de drenagem tipicamente contempladas por reservatórios de detenção são relativamente pequenas, cujo tempo de concentração costuma ser dessa ordem de grandeza. Tendo em vista o caráter expedito do processo de pré-dimensionamento ora proposto, essa hipótese pode ser preservada.

\section{Curva envelope}

0 método da "curva envelope" é bastante utilizado no dimensionamento de bacias de detenção (DAEE/CETESB, 1980). Nesse método, a curva de massa, no tempo, dos volumes afluentes é comparada com a curva de massa dos volumes efluentes, sendo a máxima diferença entre as duas curvas o volume do dimensionamento. Para o cálculo, considera-se que a curva afluente é dada pela curva PDF e a curva efluente, por simplificação do processo, é representada por uma reta, pois admite-se que a vazão de saída do dispositivo é constante. (SILVEIRA; GOLDENFUM, 2007).

Assim, definem-se as variáveis $H_{E}$ e $H_{S}$, lâminas d'água de entrada acumulada e de saída acumulada, respectivamente. $H_{E}$ é construída pela curva IDF multiplicada pelo tempo, além de fatores de escoamento e relações de área. Essa função, em mm, é dada por:

$H_{E}=\beta \cdot \frac{a \cdot T R^{b}}{(t+c)} \cdot \frac{t}{60}$ 
Dimensionamento de Reservatório de Detenção partindo da Relação IDF regente no Município de Fortaleza (CE)

Sendo $a, b$ e $c$ parâmetros da equação que dependem da área onde está sendo aplicada, $\beta$ o produto do coeficiente de escoamento pela razão entre a área contribuinte e a área do dispositivo, $t$ a duração da chuva em min e $T R$ o período de retorno em anos.

No caso de $H_{S}$, sua equação é obtida a partir da multiplicação do tempo da vazão de saída constante, conforme Equação 19:

$H_{S}=\gamma \cdot H \cdot q_{s} \cdot \frac{t}{60}$

Onde $q_{s}$ é a vazão de saída do dispositivo em $\mathrm{mm} / \mathrm{h}, \gamma$ é a razão entre a área de percolação e volume do dispositivo $\mathrm{em} \mathrm{mm}^{-1}, H$ é a profundidade média do volume de acumulação do dispositivo em mm e $t$ é a duração da chuva em min. A equação acima fornece $H_{S} \mathrm{em} \mathrm{mm}$.

\section{Obtenção do volume máximo}

0 volume máximo a ser utilizado para o dimensionamento será obtido com a maximização da diferença entre $H_{E}$ e $H_{S}$, ou seja, derivando-se o volume no tempo e igualando a zero, conforme segue:

$\frac{\partial V}{\partial t}=\frac{\partial\left(H_{E}-H_{S}\right)}{\partial t}=0$

Ilustrativamente, o procedimento da "curva envelope" é representado pela Figura 6:

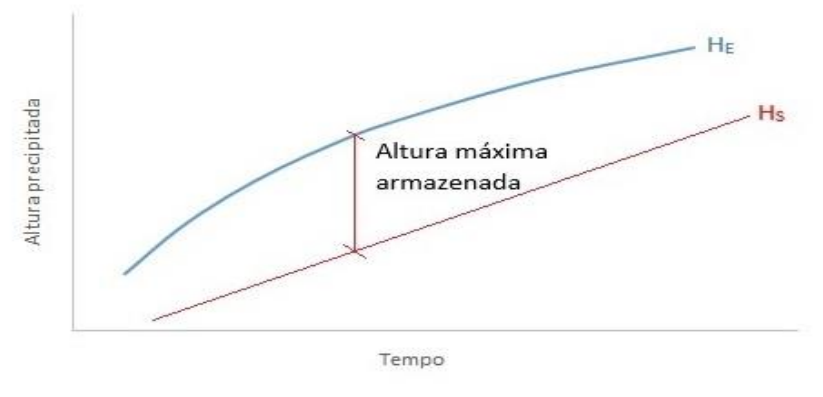

Figura 6. Metodologia da "curva envelope"

Fonte: Elaborado pelos autores, 2020.

Escrevendo $V$ como função de vazão e tempo, tem-se:

$V=\left(Q_{U}-Q_{N}\right) \cdot 60 \cdot t$

Onde $V$ é 0 volume de controle em L, $Q_{U}$ é a vazão resultante da urbanização em L/s, $Q_{N}$ é a vazão de pré-desenvolvimento em L/s e $t$ é a duração da chuva em min.

Relativizando a Equação 21 dividindo pela área, tem-se:

$v=\frac{V}{A}=\left(2,778 \cdot C \cdot i-q_{n}\right) \cdot 60 \cdot t$ 
Sendo $v$ o volume específico em L/ha.

Modificando a relação IDF de Fortaleza para compatibilizar as unidades, tem-se:

$i=\frac{6.515,22 \cdot T R^{0,173}}{(t+28,31)^{0,904}}$

A aplicação da Equação 23 fornece $i$ em L/s/ha.

Substituindo as Equações 16 e 23 na Equação 22 e considerando $q_{n}=25,3 \mathrm{~L} / \mathrm{s} / \mathrm{ha}$, resulta-se em:

$v=\frac{60}{1000} \cdot t \cdot\left[\frac{(0,15+0,80 \cdot A I) \cdot 6.515,22 \cdot T R^{0,173}}{(t+28,31)^{0,904}}-25,3\right]$

Sendo $v \mathrm{em} \mathrm{m}^{3} /$ ha e $t$ em min.

Aplicando, na Equação 24, a derivada em relação ao tempo, conforme descrito no procedimento da “curva envelope", resulta-se em:

$\frac{\partial v}{\partial t}=0,06 \cdot t \cdot\left[\frac{\beta}{(t+28,31)^{0,904}}-25,3\right]-0,06 \cdot t \cdot \frac{\beta \cdot 0,904 \cdot(t+28,31)^{-0,096}}{(t+28,31)^{1,808}}$

Sendo:

$\beta=(0,15+0,80 \cdot A I) \cdot 6.515,22 \cdot T R^{0,173}$

Igualando a Equação 25 a zero, resulta-se em:

$\beta \cdot(t+28,31)-25,3 \cdot(t+28,31)^{1,904}-t \cdot \beta \cdot 0,904=0$

A solução da Equação 27, ou seja, a obtenção do tempo de duração, permite estimar o volume específico pela aplicação desse tempo na Equação 24.

\section{RESULTADOS E DISCUSSÃ0}

Aplicando a Equação 17 em variados períodos de retorno, resulta-se na Tabela 1 a seguir: Tabela 1 - Vazões específicas de pré-dimensionamento

\begin{tabular}{c|c|c}
\hline TR (anos) & $\mathrm{i}(\mathrm{mm} / \mathrm{h})$ & $\mathrm{q}_{\mathrm{n}}(\mathrm{L} / \mathrm{s} / \mathrm{ha})$ \\
\hline 2 & 46,0 & 19,2 \\
\hline 5 & 53,9 & 22,5 \\
\hline 10 & 60,8 & 25,3 \\
\hline 25 & 71,3 & 29,7 \\
\hline 50 & 80,3 & 33,5 \\
\hline 100 & 90,6 & 37,7 \\
\hline
\end{tabular}

Fonte: Elaborado pelos autores, 2020.

No âmbito deste trabalho, foi adotado, para fins de estimativa de dimensionamento, o valor de $T R=10$ anos e, portanto, $q_{n}=25,3 \mathrm{~L} / \mathrm{s} / \mathrm{ha}$. Neste estudo, $q_{n}$ está sendo considerada a vazão natural, com a recorrência do evento crítico tipicamente assumida nos sistemas 
Dimensionamento de Reservatório de Detenção partindo da Relação IDF regente no Município de Fortaleza (CE)

convencionais de microdrenagem. Poderia ser também considerada como a capacidade de drenagem na área onde será projetado novo desenvolvimento, ou seja, uma expansão urbana.

\section{Obtenção dos tempos de duração}

Com 10 diferentes porcentagens de área impermeável e 6 diferentes períodos de retorno, resulta-se em 60 combinações e 60 valores a serem determinados para tempo de duração. Para tanto, foi utilizado o software MATLABß (MATrix LABoratory) para realizar as iterações da Equação 28 a seguir e encontrar os valores de $t$ que a satisfazem e resultar na Tabela 2.

$(0,15+0,80 \cdot A I) \cdot 6.515,22 \cdot T R^{0,173} \cdot(t+28,31)-25,3 \cdot(t+28,31)^{1,904}-t \cdot(0,15+$ $0,80 \cdot A I) \cdot 6.515,22 \cdot T R^{0,173} \cdot 0,904=0$

Tabela 2 - Tempos de duração em min para as combinações de \%AI e TR

\begin{tabular}{c|c|c|c|c|c|c}
\hline \multirow{2}{*}{$\begin{array}{c}\text { \% Área } \\
\text { Impermeável }\end{array}$} & \multicolumn{7}{|c}{ Período de retorno (anos) } \\
\cline { 2 - 7 } & 2 & 5 & 10 & 25 & 50 & 100 \\
\hline 10 & 26,729 & 32,022 & 36,394 & 42,713 & 47,943 & 53,601 \\
\hline 20 & 37,159 & 43,560 & 48,859 & 56,537 & 62,910 & 69,821 \\
\hline 30 & 46,650 & 54,084 & 60,251 & 69,207 & 76,657 & 84,754 \\
\hline 40 & 55,484 & 63,901 & 70,896 & 81,075 & 89,559 & 98,796 \\
\hline 50 & 63,827 & 73,190 & 80,985 & 92,346 & 101,833 & 112,180 \\
\hline 60 & 71,785 & 82,067 & 90,638 & 103,153 & 113,620 & 125,054 \\
\hline 70 & 79,433 & 90,611 & 99,943 & 113,587 & 125,017 & 137,520 \\
\hline 80 & 86,824 & 98,880 & 108,958 & 123,714 & 136,094 & 149,653 \\
\hline 90 & 93,997 & 106.917 & 117,730 & 133,583 & 146,902 & 161,506 \\
\hline 100 & 100,984 & 114,756 & 126,295 & 143,234 & 157,482 & 173,124 \\
\hline
\end{tabular}

Fonte: Elaborado pelos autores, 2020.

Para qualquer valor de $T R$ e \%AI da tabela anterior, a vazão de pré-dimensionamento refere-se a 10 anos, ou seja, 25,3 L/s/ha, correspondente ao nível que se supõe aceitável no impacto da urbanização. Nota-se que o valor de tempo de duração é tanto maior quanto a porcentagem de área impermeável e o período de retorno.

\section{Obtenção das alturas de lâmina d'água}

A Tabela 3 foi elaborada substituindo os valores de $t, T R$ e $\% A I$ da Tabela 2 na Equação 24.

Tabela 3 - Volumes específicos em $\mathrm{m}^{3}$ /ha para as combinações de \% AI e TR

\begin{tabular}{|c|c|}
\hline $\begin{array}{c}\text { \% Área } \\
\text { Impermeável }\end{array}$ & Período de retorno (anos) \\
\hline
\end{tabular}


Fonte: Elaborado pelos autores, 2020.

\begin{tabular}{c|c|c|c|c|c|c}
\hline & 2 & 5 & 10 & 25 & 50 & 100 \\
\hline 10 & 31,753 & 44,837 & 57,151 & 77,246 & 95,837 & 117,848 \\
\hline 20 & 59,440 & 80,138 & 99,268 & 130,016 & 158,110 & 191,066 \\
\hline 30 & 91,079 & 119,814 & 146,115 & 188,040 & 226,077 & 270,460 \\
\hline 40 & 125,592 & 162,682 & 196,419 & 249,913 & 298,224 & 354,400 \\
\hline 50 & 162,336 & 208,030 & 249,416 & 314,791 & 373,640 & 441,902 \\
\hline 60 & 200,886 & 255,392 & 304,601 & 382,115 & 451,723 & 532,313 \\
\hline 70 & 240,946 & 304,438 & 361,618 & 451,492 & 532,047 & 625,174 \\
\hline 80 & 282,294 & 354,924 & 420,206 & 522,632 & 614,296 & 720,143 \\
\hline 90 & 324,763 & 406,667 & 480,163 & 595,312 & 698,231 & 816,958 \\
\hline 100 & 368,223 & 459,519 & 541,332 & 669,356 & 783,661 & 915,415 \\
\hline
\end{tabular}

Assim como na Tabela 2, os valores de volume específico crescem à medida que também crescem a porcentagem de área impermeável e o período de retorno. Os valores da Tabela 3 estão expostos no gráfico da Figura 7:

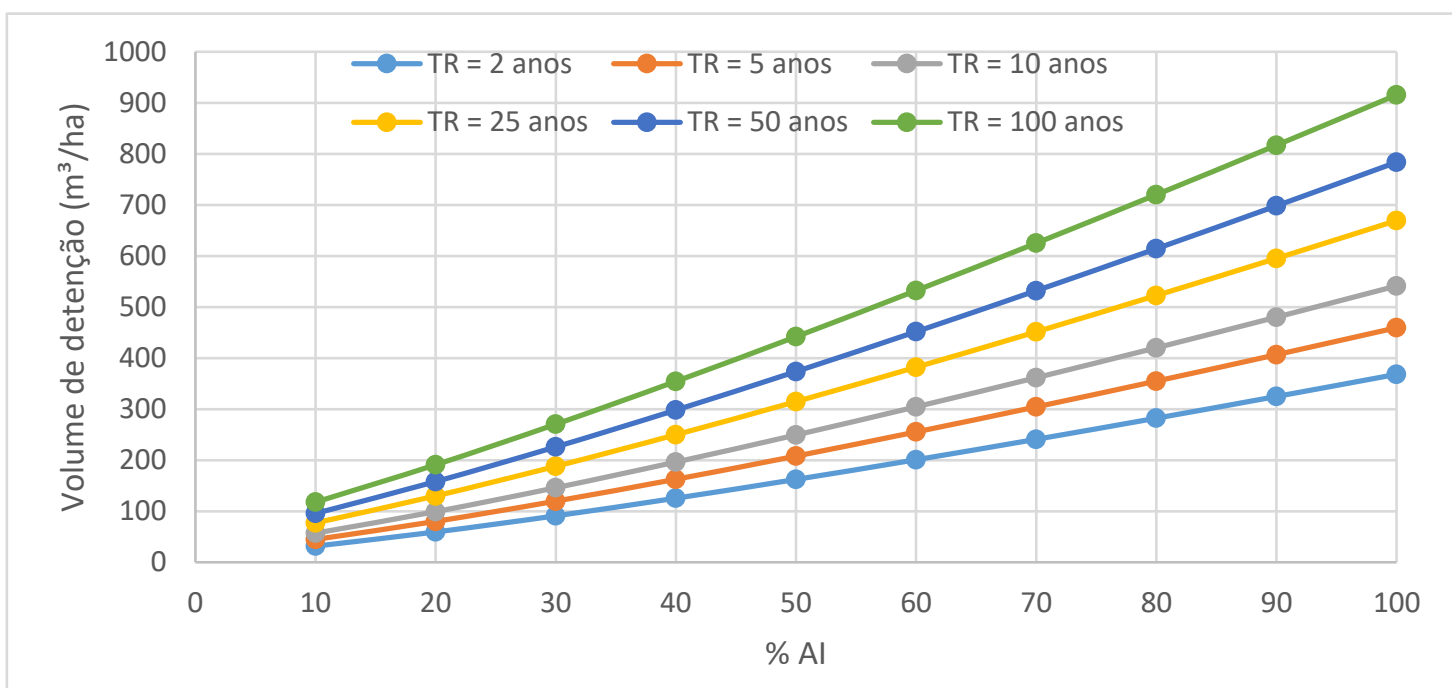

Figura 7. Valores de volumes específicos em função da \%AI para cada período de retorno Fonte: Elaborado pelos autores, 2020.

Pelo gráfico, pode-se estimar o volume que irá servir como base para o dimensionamento do reservatório de detenção. Por exemplo, para uma área de contribuição de 40 hectares, área impermeável de $70 \%$ e período de retorno de 25 anos, o reservatório de detenção necessitará dispor de 18.059,68 $\mathrm{m}^{3}$ de capacidade para uma vazão de pré-dimensionamento de 25,3 L/s/ha.

\section{Equação de ajuste}

A partir da Tabela 3, foi executado um procedimento matemático de regressão no MATLAB® para elaborar uma equação de ajuste que fornece valores suficientemente próximos. 
Dimensionamento de Reservatório de Detenção partindo da Relação IDF regente no Município de Fortaleza (CE)

Para tanto, entende-se que o volume específico é uma função de $T R$ e \%AI. Foram montadas duas equações a partir desse procedimento, sendo a primeira com abrangência para os períodos de retorno de 2, 5, 10 e 25 anos, denominada Equação 29, e a segunda abrangendo todos os períodos de retorno utilizados neste trabalho, denominada Equação 30.

$v=2,6072 \cdot T R^{0,27067} \cdot A I^{1,0146}$

$v=2,9802 \cdot T R^{0,26302} \cdot A I^{0,98281}$

Na Figura 8 é ilustrada a aplicação das duas equações acima e sua comparação com os valores obtidos pelo MATLAB® para o período de retorno de 25 anos.

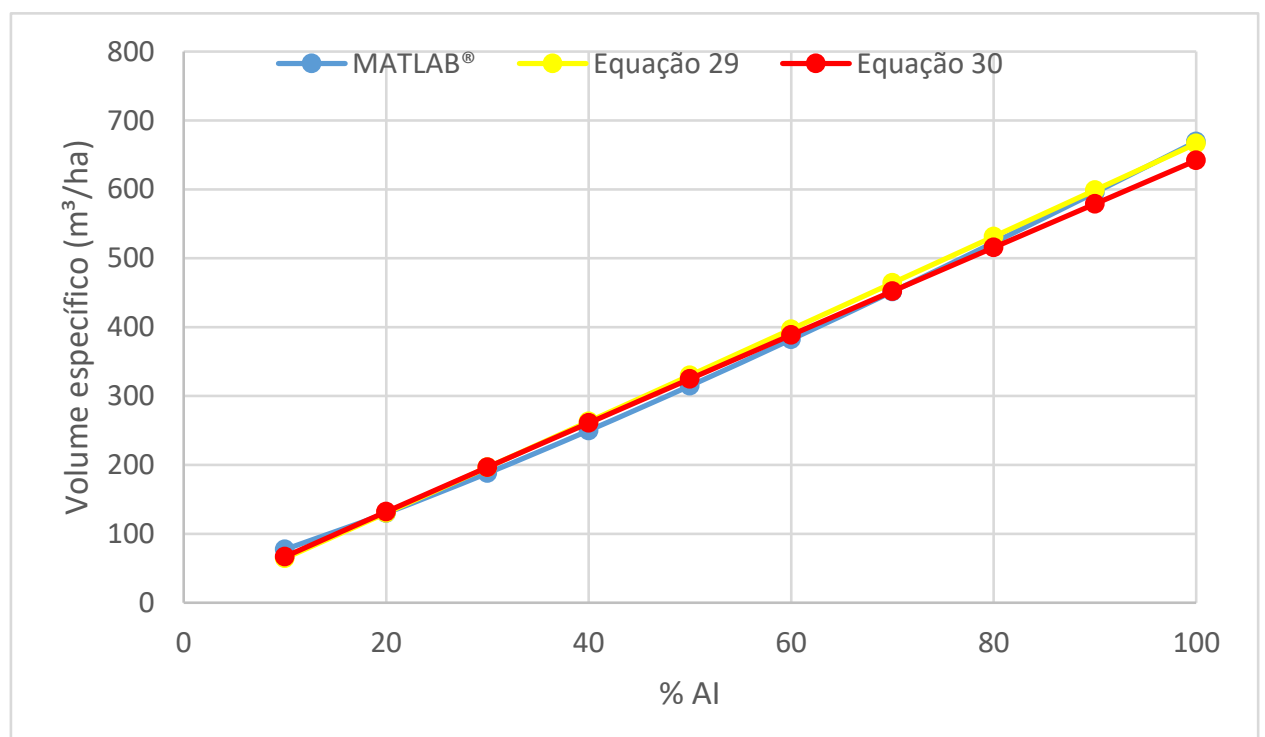

Figura 8. Comparação entre os valores obtidos pelo MATLABß e pelas Equações 29 e 30 Fonte: Elaborado pelos autores, 2020.

Em termos estatísticos, aplicando a Equação 29 nos períodos de retorno de 2 a 25 anos e a Equação 30 nos períodos de retorno de 2 a 100 anos, geram-se, respectivamente, 40 e 60 pontos de aplicação. Para avaliar a qualidade desses ajustes, apurou-se como medida de dispersão a raiz quadrada do erro relativo quadrático médio em relação àqueles valores mostrados na Tabela 3. Esse parâmetro de dispersão revelou valores de 0,055 e 0,062, respectivamente, para as Equações 29 e 30, satisfatórios para o propósito do trabalho, contemplando a fase de planejamento das intervenções.

\section{CONCLUSÃ0}

Enfatiza-se aqui a necessidade de se dimensionar dispositivos de atenuação de enchentes abordando estratégias de técnicas compensatórias, sendo importante realizar 
estudos prévios principalmente na fase de planejamento do sistema de manejo de águas pluviais.

A metodologia implementada permite que se disponibilize ao planejador uma estratégia de pré-dimensionamento, com as características da chuva local, para contemplar aspectos hidrológicos importantes no processo de gestão do uso e ocupação do solo e, assim, estimular uma intervenção no manejo de águas pluviais de forma a atenuar os impactos da urbanização na bacia hidrográfica a partir da implantação de medida compensatória pautada no armazenamento temporário da água excedente.

A vantagem da formulação mostrada neste trabalho se evidencia pela possibilidade de aplicação em diversas áreas do município de Fortaleza, podendo-se variar as taxas ocupacionais e períodos de retorno de acordo com o interesse do projetista em dimensionar reservatórios de detenção para determinado bairro ou considerando futura ampliação.

\section{REFERÊNCIAS BIBLIOGRÁFICAS}

ABCP. Projeto Técnico: Reservatórios de Detenção. São Paulo: Soluções Para Cidades, 2013. Disponível em: http://solucoesparacidades.com.br/saneamento/reservatorios-de-detencao/. Acesso em: 25 abr. 2020.

BAHIENSE, Juliana Martins. Avaliação de Técnicas Compensatórias em Drenagem Urbana Baseadas no Conceito de Desenvolvimento de Baixo Impacto, com o Apoio de Modelagem Matemática. 2013. 135 f. Dissertação (Mestrado) Curso de Engenharia Civil, Universidade Federal do Rio de Janeiro, Rio de Janeiro, 2013. Disponível em: http://www.coc.ufrj.br/pt/documents2/mestrado/2013/2518-juliana-martins-bahiense-mestrado .Acesso em: 25 abr. 2020 .

BAPTISTA, Márcio; NASCIMENT0, Nilo; BARRAUD, Sylvie. Técnicas Compensatórias em Drenagem Urbana. Porto Alegre: ABRH, 2005. 318 p.

BIDONE, Francisco R. A.; TUCCI, Carlos E. M.. Microdrenagem. In: TUCCI, Carlos E. M. (Org.); P0RT0, Rubem La Laina (Org.); BARROS, Mário T. de (Org.). Drenagem Urbana. 1. ed. Porto Alegre: Editora da Universidade, 1995. Cap. 3. p.77 105.

DAEE/CETESB. 1980. Drenagem Urbana: Manual de Projeto. Departamento de Águas e Energia Elétrica e Companhia de Tecnologia de Saneamento. São Paulo.

MIGUEZ, Marcelo Gomes; VERÓL, Aline Pires; REZENDE, Osvaldo Moura. Drenagem Urbana: Do projeto tradicional à sustentabilidade. 1. ed. Rio de Janeiro: Elsevier Editora Ltda., 2015. 352 p.

SCHUELER, T. R., 1987. Controlling Urban Runoff: A Practical Manual for Planning and Designing Urban BMPs. Department of Environmental Programs, Metropolitan Washington Council of Governments.

SECRETARIA NACIONAL DE SANEAMENTO AMBIENTAL. Águas Pluviais: Técnicas Compensatórias para o Controle de Cheias Urbanas. Belo Horizonte: ReCESA, 2007. 52 p. Disponível em: https://www.mdr.gov.br/images/stories/ArquivosSNSA/Arquivos_PDF/recesa/tecnicascompensatoriasparaocontr oledecheiasurbanas-nivel2e3.pdf. Acesso em: 21 abr. 2020.

SILVA, Francisco Osny Enéas da; PALÁCIO JUNIOR, Francisco Flávio Rocha; CAMPOS, José Nilson Bezerra. Equação de chuvas para Fortaleza CE com dados do pluviógrafo da UFC. Revista DAE, Fortaleza, v. 1491, n. 192, p.48 59, 18 dez. 2012. Disponível em: http://revistadae.com.br/artigos/artigo_edicao_192_n_1491.pdf. Acesso em: 07 abr. 2020. 
Dimensionamento de Reservatório de Detenção partindo da Relação IDF regente no Município de Fortaleza (CE)

SILVEIRA, André Luiz Lopes da; GOLDENFUM, Joel Avruch. Metodologia Generalizada para Pré-Dimensionamento de Dispositivos de Controle Pluvial na Fonte. Revista Brasileira de Recursos Hídricos, Porto Alegre, v. 12, n. 2, p. 157-168, 02 abr. 2007. Disponível em: https://wp.ufpel.edu.br/hugoguedes/files/2018/09/Silveira-e-Goldengum-2007RBRH-v.12-n.2-2007.pdf.Acesso em: 21 abr. 2020.

SOUZA, Felipe Miranda; MEDEIROS, Carlos Luciano Rodrigues; MIRANDA, Daniel Augusto de. Proposição de técnicas compensatórias como alternativa para controle das águas escoadas no empreendimento imobiliário Fashion City (Pedro Leopoldo/MG). Revista Petra, Belo Horizonte, v. 3, n. 2, p. 121-141, dez. 2017. Disponível em: https://www.metodista.br/revistas/revistas-izabela/index.php/ptr/article/view/1l23. Acesso em: 25 abr. 2020.

SUPERINTENDÊNCIA DE DESENVOLVIMENTO DE RECURSOS HÍDRICOS E SANEAMENTO AMBIENTAL (Paraná). Manual de drenagem urbana da região metropolitana de Curitiba. Curitiba: SUDERHSA, 2002. Disponível em http://www.aguasparana.pr.gov.br/arquivos/File/pddrenagem/volume6/mdu_versa001.pdf.Acesso em: 09 abr. 2020.

TEIXEIRA, Lucas Florêncio da Cunha. Avaliação do Desempenho Hidráulico de Sarjetas no Sistema de Drenagem de Fortaleza. 2017. 66 f. TCC (Graduação) - Curso de Engenharia Civil, Universidade de Fortaleza, Fortaleza, 2017.

THE MATHWORKS. MATLAB®. 2020. Natick, Massachusetts.

TUCCI, Carlos E. M. Vazão Máxima e Hidrograma de Projeto. In: TUCCI, Carlos E. M. (Org.). Hidrologia: Ciência e Aplicação. 1. ed. Porto Alegre: Editora da Universidade, 1993. Cap. 14. p.527-572. 